\title{
Gastric cancer initially presenting as bone metastasis: Two case reports and a literature review
}

\author{
DAISUKE GOMI, TOSHIROU FUKUSHIMA, TAKASHI KOBAYASHI, NODOKA SEKIGUCHI, \\ AKIYUKI SAKAMOTO, KEIKO MAMIYA and TOMONOBU KOIZUMI
}

Department of Comprehensive Cancer Therapy, Shinshu University School of Medicine, Matsumoto, Nagano 390-8621, Japan

Received September 14, 2017; Accepted April 16, 2018

DOI: $10.3892 / 01.2018 .9393$

\begin{abstract}
Gastric cancer frequently spreads to the regional lymph nodes, liver and lungs following surgery or late in the clinical course. However, an initial clinical presentation of bone metastasis in gastric cancer patients is relatively rare. The current study presents two cases of gastric cancer diffusely metastasized to the spinal vertebrae and with a single metastasis to the trapezium, respectively. The initial presentations were an increased alkaline phosphatase level without any symptoms associated with bone metastasis in the first case and a swelling in the right carpometacarpal joint of the thumb in the second case. These clinical manifestations are also extremely rare in gastric cancer with bone metastasis. The study emphasizes that a diagnosis of gastric cancer should be considered in patients with increased alkaline phosphatase without clinical symptoms or with a single bone metastasis.
\end{abstract}

\section{Introduction}

Gastric cancer frequently spreads to the regional lymph nodes, liver and lungs following surgery or late in the clinical course (1). Gastric cancer rarely shows dissemination to the bones, and the incidence of bone metastasis due to gastric cancer is only $0.9-3.8 \%$ (1-4). Bone metastasis is usually recognized as parallel disease recurrence following surgery (1-3) or progression during follow-up, and occurs in the late stage of the disease (2-4). Initial (or simultaneous) presentation of bone metastasis, particularly in asymptomatic gastric cancer, is extremely rare (5-8). The present study describes two cases of gastric cancer initially presenting with an increased serum alkaline phosphatase (ALP) level without any clinical symptoms and as a swelling in the right carpometacarpal joint of

Correspondence to: Professor Tomonobu Koizumi, Department of Comprehensive Cancer Therapy, Shinshu University School of Medicine, 3-1-1 Asahi, Matsumoto, Nagano 390-8621, Japan E-mail: tomonobu@shinshu-u.ac.jp

Key words: cisplatin, S-1, chemotherapy, disseminated bone metastasis, single bone metastasis, trapezium the thumb, respectively. The former case showed diffuse spinal vertebral bone metastasis, while the latter showed a single trapezium metastasis. The two cases were diagnosed as metastatic gastric cancer on further examination. The present study also describes the clinical courses and reviews the initial bone metastases in patients with gastric cancer from the literature.

\section{Case report}

Case 1. A 60-year-old woman with no significant past medical history or symptoms was referred to Shinshu University Hospital (Nagano, Japan) on April 2013 due to progressive increases in ALP level that had been detected by routine health screening over a period of 7 months. Physical examinations were unremarkable. Laboratory findings indicated a markedly increased ALP level (4,917 IU/l; normal, <322 IU/l), but other findings were normal, including the level of carcinoembryonic antigen (CEA; $1.9 \mathrm{mg} / \mathrm{dl}$; normal, <0.5 mg/dl). Bone scintigraphy showed multiple abnormal uptake of radioactive material particularly in the lumbar vertebrae (Fig. 1A). A review of computed tomography (CT) (Fig. 1B) and magnetic resonance imaging data showed multiple osteolytic bone metastases, particularly in the lumbar vertebrae. Fluorodeoxyglucose-positron emission tomography/CT (FDG-PET/CT) revealed multiple bone metastases, including pubic, rib and lumbar vertebrae metastases, with no other abnormal FDG uptake except in these bone lesions. Needle biopsy of the left iliac bone was performed and the histological findings showed adenocarcinoma with the formation of large vacuoles full of mucin that displace the nucleus to the cell's periphery suggesting signet cell carcinoma (Fig. 2A and B). The specimen was fixed in $20 \%$ neutral-buffered formalin and decalcified in $10 \%$ formic acid for one day at room temperature. The decalcified specimen block was then embedded in paraffin, sliced into sections of 4- $\mu \mathrm{m}$ thickness, and stained with hematoxylin and eosin for $90 \mathrm{~min}$ at room temperature. The images were captured with an Olympus BX51 microscope (objective, x40) and Olympus DP70 camera. Histological findings (Fig. 2C and D) of a small flat depressed lesion in the middle gastric body area, which was revealed by esophagogastroduodenoscopy (Fig. 3), revealed the presence of similar signet cell carcinoma in gastric mucosa with those in iliac bone as aforementioned. Therefore, a diagnosis of gastric signet cell carcinoma with multiple bone metastases was 

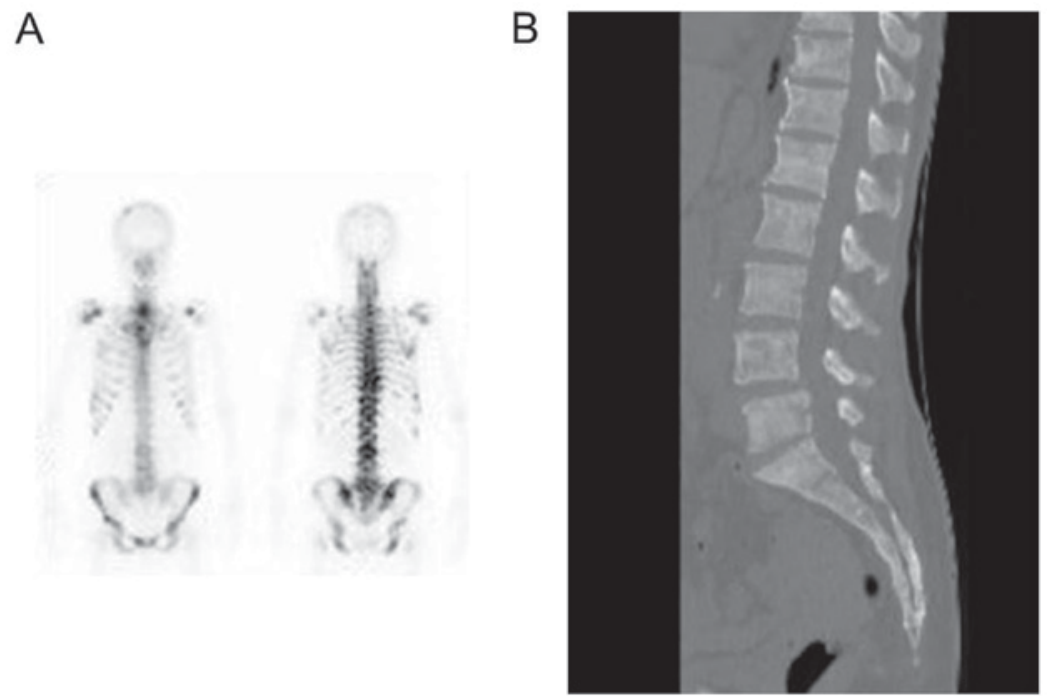

Figure 1. Case 1: (A) Bone scintigraphy showed multiple abnormal uptake of radioactive material, particularly in the lumbar vertebrae, and (B) computed tomography showed multiple osteolytic bone metastases, particularly in the lumbar vertebrae.

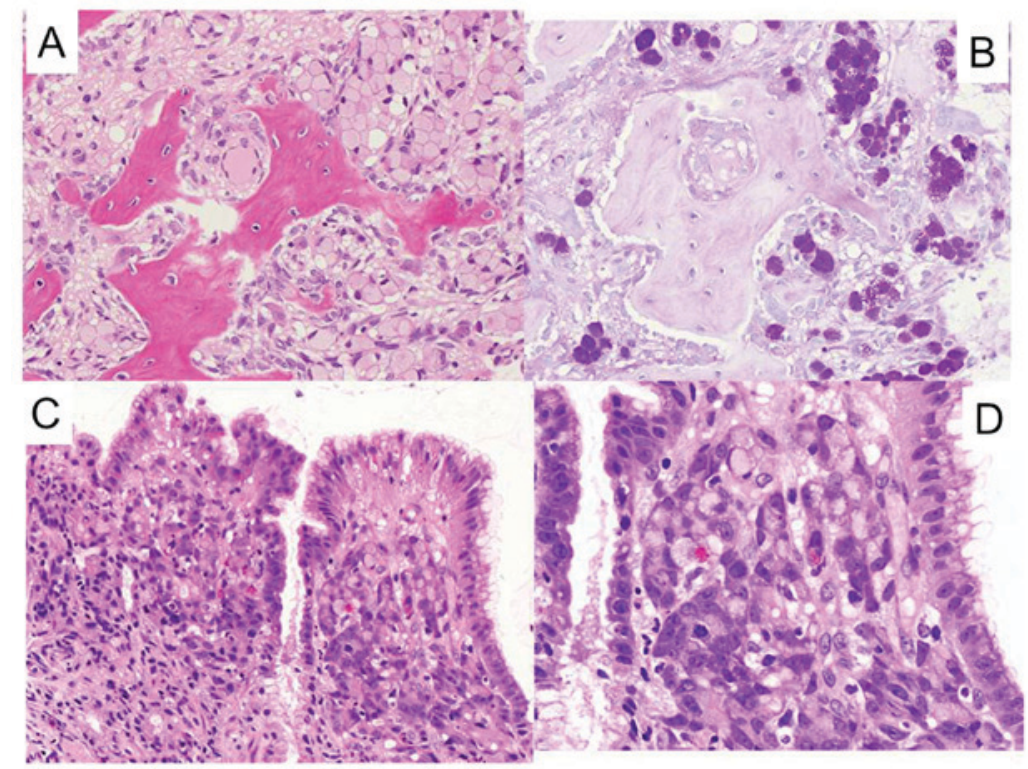

Figure 2. Case 1: (A and B) Needle biopsy specimens from the left iliac bone and (C and D) endoscopic biopsy from the gastric body area, obtained by esophagogastroduodenoscopy, revealed signet-ring cell carcinoma. (A) H\&E magnification, x40; (B) periodic acid-Schiff magnification, x40; (C) H\&E magnification, x40; (D) H\&E magnification, x100. H\&E, hematoxylin and eosin.

made. Immunohistochemical analysis for epidermal growth factor receptor 2 (HER2) was negative in this case. The patient received combined chemotherapy of cisplatin $\left(80 \mathrm{mg} / \mathrm{m}^{2}\right.$, day 8 postoperatively) plus $\mathrm{S}-1\left(80 \mathrm{mg} / \mathrm{m}^{2}\right.$, day $1-21$ postoperatively) followed by S-1 monotherapy $\left(80 \mathrm{mg} / \mathrm{m}^{2}\right.$, day $1-28$ every 6 weeks), and denosumab (a fully human monoclonal antibody that inhibits receptor activator of nuclear factor- $\kappa \mathrm{B}$ ligand, $120 \mathrm{mg}$ once every 6 weeks). Subsequent to six cycles of cisplatin plus S-1 therapy, gastrointestinal endoscopic findings confirmed scarring of the primary gastric cancer, and the ALP was determined to have returned to a normal level (189 IU/l). The disease was controlled with S-1 monotherapy over 1 year, but the patient finally developed disseminated intravascular coagulation (DIC) and liver metastasis, and succumbed 2 years after diagnosis.
Case 2. A 62-year-old man presented with a swelling in the right carpometacarpal (CMC) joint of the thumb. Conservative treatment for thumb CMC osteoarthritis had been performed for 3 months. However, radiographic findings revealed an osteolytic lesion in the trapezium, with resorption of almost the whole of the trapezium (Fig. 4A) and referred to Shinshu University Hospital on October 2014. Needle biopsy was performed and histopathological examination was performed as described previously, and indicated the presence of tubular adenocarcinoma. FDG-PET/CT revealed positive accumulation of FDG in the trapezium and epigastrium (Fig. 4B). Based on the results of FDG-PET, an esophagogastroduodenoscopy was performed and showed an advanced and ulcerative tumor (Fig. 5A). The results of histological examination of the gastric specimens were consistent with those in the trapezium as 


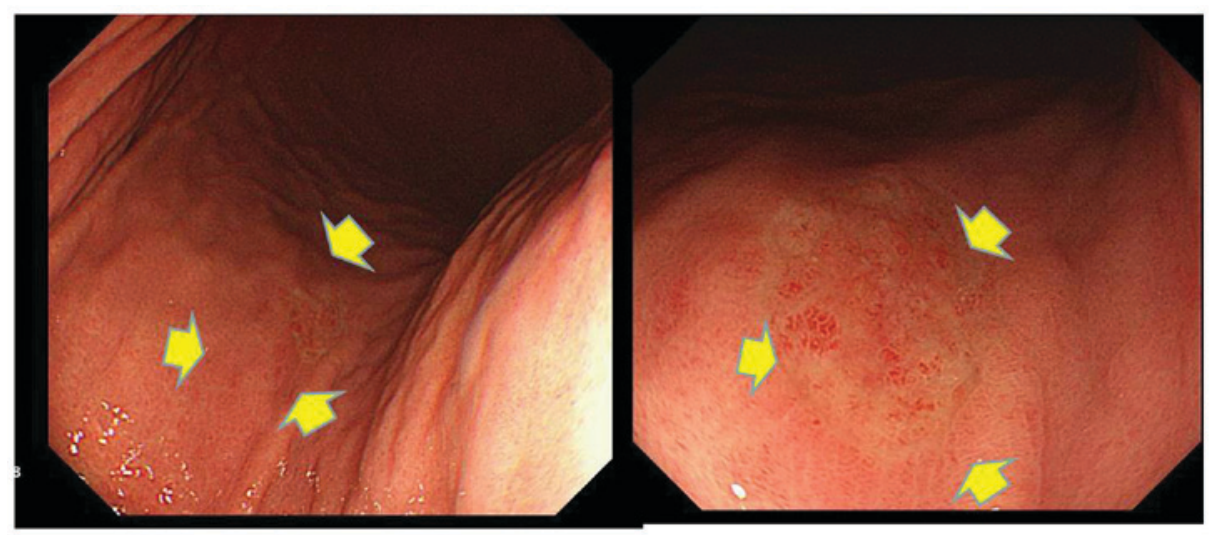

Figure 3. Case 1: Esophagogastroduodenoscopic findings revealed a small flat depressed lesion in the middle gastric body area. The arrows indicate the lesion.
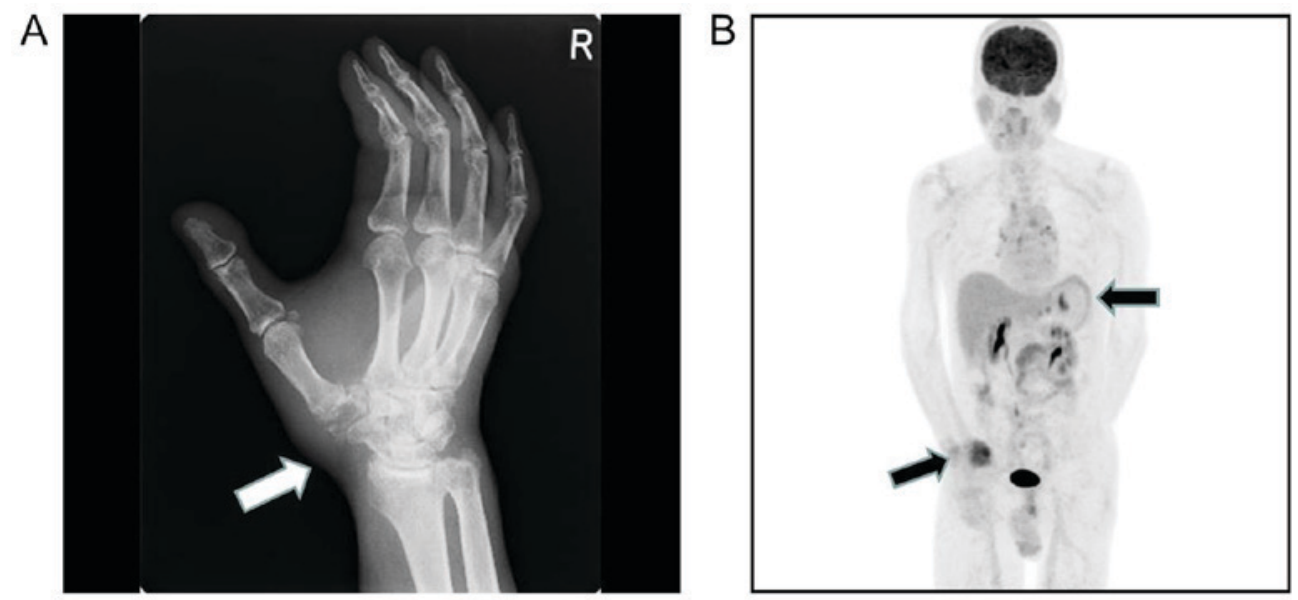

Figure 4. Case 2: (A) Radiography revealed an osteolytic lesion in the trapezium with resorption of almost the whole trapezium (arrow indicates the osteolytic lesion). (B) FDG-positron emission tomography/computed tomography revealed positive accumulation of FDG in the trapezium and epigastrium (arrows indicate the positive lesions). FDG, fluorodeoxyglucose.
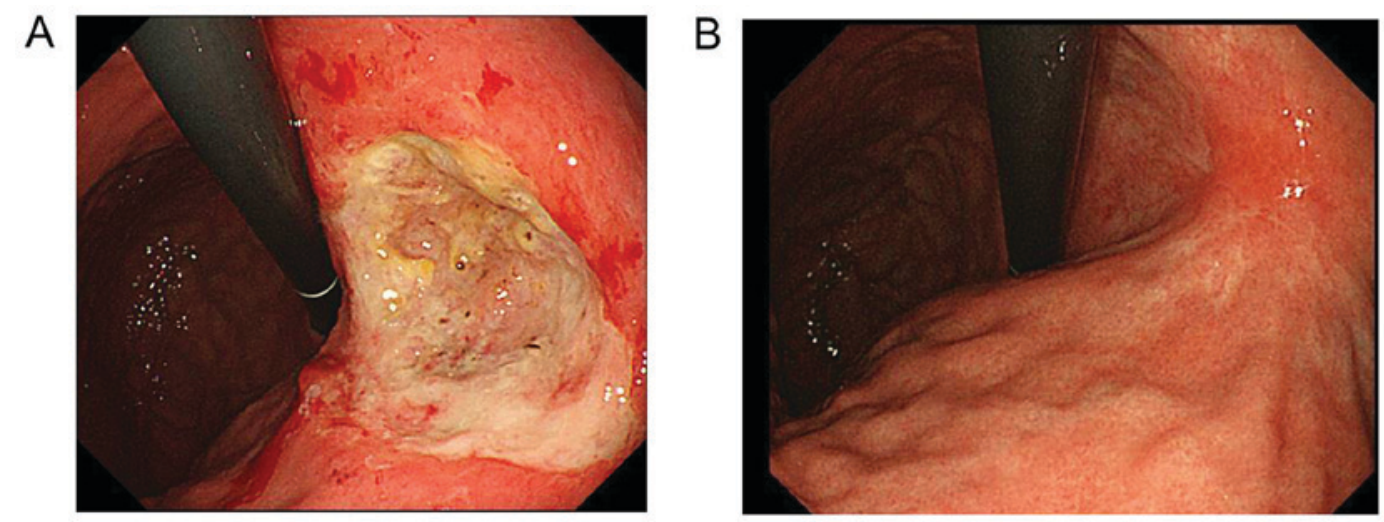

Figure 5. Case 2: Esophagogastroduodenoscopic findings (A) prior to and (B) subsequent to six cycles of cisplatin plus S-1 chemotherapy. The advanced and ulcerative tumor disappeared almost completely following chemotherapy.

aforementioned. The serum CEA level was normal $(3.2 \mathrm{mg} / \mathrm{ml})$. No other distant metastases were found. Therefore, a diagnosis of advanced gastric adenocarcinoma with a single metastasis to the CMC joint of the thumb was made. Immunohistochemical analysis for HER2 was negative in this case. Radiotherapy (3 Gy/10 fractions; total, $30 \mathrm{~Gy}$ ) to the metastatic CMC area was performed. The patient was treated with six cycles of systemic chemotherapy with cisplatin $\left(60 \mathrm{mg} / \mathrm{m}^{2}\right.$ day 8$)$ and S-1 (80 mg $/ \mathrm{m}^{2}$ day $\left.1-21\right)$, followed by $\mathrm{S}-1$ ( $80 \mathrm{mg} / \mathrm{m}^{2}$, day $1-28$, every 6 weeks) maintenance therapy, which resulted in a partial response of the primary lesion to the chemotherapy (Fig. 5B). The patient visited Shinshu University every 6 weeks for S-1 maintenance therapy and remains alive 2 years after the initiation of chemotherapy without any signs of recurrence. 


\section{Discussion}

According to 3 case reports, initial clinical manifestations associated with bone metastasis are extremely rare in patients with gastric cancer (6-8). In the present study, increased ALP levels were noted by routine laboratory survey in case 1 , without any symptoms associated with bone involvement, and swelling in the right $\mathrm{CMC}$ joint of the thumb due to trapezium metastasis was noted in case 2 . The clinical manifestations of bone metastasis observed in these cases are extremely rare as the initial signs of asymptomatic gastric cancer. Clinical physicians should be aware of the possibility of initial bone involvement in patients with gastric cancer.

Kim et al (5) summarized the findings in patients with initial bone metastasis in gastric cancer and reported that bone metastasis is often accompanied by lymphangitic lung metastasis, pleural metastasis, leptomeningeal metastasis, a primary tumor involving more than two-thirds of the stomach and hematological abnormalities, including DIC or elevated ALP. In addition, the most common histological type was signet cell carcinoma. ALP is known to be the most predictive biological marker for the presence of bone metastasis in gastric cancer (9). In the present study, in case 1, the signet cell carcinoma histological type and elevated level of ALP were consistent with these predictors of initial bone metastasis in gastric cancer. However, the patient was asymptomatic and presented with no other metastatic sites. In addition, endoscopic findings indicated a small abnormality so-called occult primary tumor in case 1 . These cases suggested that gastric cancer should be considered in patients with elevated ALP without any clinical symptoms. A search for similar case reports in PubMed (https://www.ncbi. nlm.nih.gov/pubmed/) using the terms 'occult gastric cancer', 'bone metastasis' or 'ALP' did not find any similar cases. Thus, although the clinical manifestations in case 1 were quite unique, it should be emphasized that gastric cancer is a possible disease in patients with elevated ALP.

The most frequent sites of bone metastasis are the vertebrae, followed by the ribs, scapulae, lower extremities and upper extremities (10-12). In the present study, the location and single metastasis to the trapezium in case 2 is noteworthy. To the best of our knowledge, this is the first report of a single metastasis from gastric cancer to the trapezium. Although endoscopic findings of the primary lesion showed advanced gastric cancer, a single trapezium metastasis without any other distant spread is extremely rare in gastric cancer.

Riihimäki et al (13) reported that gastric cancer patients with bone metastasis showed the poorest median survival time compared with those with metastases in other sites, including the thorax, liver or other regions of the abdomen. In addition, patients with initial (simultaneous) bone metastasis in gastric cancer exhibited significantly poorer overall survival time following the diagnosis of gastric cancer compared with late-phase bone metastasis $(3,5,10)$. However, survival time following diagnosis of bone metastasis in gastric cancer was slightly longer in the initial bone metastasis patients compared with that in those with late-phase bone metastasis (5). Several studies indicated that systemic chemotherapy could improve survival, even in patients with initial bone metastasis of gastric cancer (3-5,14). In the two cases presented in the present study, cisplatin plus S-1, a standard chemotherapy regimen for advanced gastric cancer (15), was effective for disease control. Subsequent to chemotherapy, the ALP level returned to normal in case 1 and the gastric tumor was undetectable on endoscopy in case 2, which may indicate a favorable clinical outcome in each case. Thus, these results suggest that cisplatin plus S-1 is a useful chemotherapeutic regimen for gastric cancer with bone metastasis.

In summary, the present cases indicated that gastric cancer exhibits a variety of clinical features with regard to bone metastasis, particularly in the initial clinical presentation of the disease. In addition, the cases indicated the importance of the detection of potential gastric cancer in patients with an initial presentation of bone metastasis. Thus, gastric cancer should be considered in the differential diagnosis in patients initially presenting with single or multiple bone metastases.

\section{Acknowledgements}

Not applicable.

\section{Funding}

No funding was received for the present case study.

\section{Availability of data and materials}

The datasets used and/or analyzed during the current study are available from the corresponding author on reasonable request.

\section{Authors' contributions}

DG, TF, TK, NS, AS, KM and TK contributed to the treatment of the two cases, and DG and TK wrote the manuscript.

\section{Ethics approval and consent to participate}

Written informed consent for the publication of any associated data and accompanying images was obtained from the patients or their guardians.

\section{Patient consent for publication}

Written informed consent for the publication of any associated data and accompanying images was obtained from the patients or their guardians.

\section{Competing interests}

The authors declare that they have no competing interests.

\section{References}

1. Guadagni S, Catarci M, Kinoshitá T, Valenti M, De Bernardinis $\mathrm{G}$ and Carboni $\mathrm{M}$ : Causes of death and recurrence after surgery for early gastric cancer. World J Surg 21: 434-439, 1997.

2. Nakamura K, Tomioku M, Nabeshima K and Yasuda S: Clinicopathologic features and clinical outcomes of gastric cancer patients with bone metastasis. Tokai J Exp Clin Med 39: 193-198, 2014.

3. Turkoz FP, Solak M, Kilickap S, Ulas A, Esbah O, Oksuzoglu B and Yalcin S: Bone metastasis from gastric cancer: The incidence, clinicopathological features, and influence on survival. J Gastric Cancer 14: 164-172, 2014. 
4. Park HS, Rha SY, Kim HS, Hyung WJ, Park JS, Chung HC, Noh SH and Jeung HC: A prognostic model to predict clinical outcome in gastric cancer patients with bone metastasis. Oncology 80: 142-150, 2011

5. Kim YJ, Kim SH, Kim JW, Lee JO, Kim JH, Bang SM, Lee JS and Lee KW: Gastric cancer with initial bone metastasis: A distinct group of diseases with poor prognosis. Eur J Cancer 50: 2810-2821, 2014

6. Basheer A, Daniel J and Padhi S: Compressive myeloradiculopathy from bony metastasis as the initial presentation of poorly differentiated adenocarcinoma stomach-a case report. Australas Med J 6: 515-519, 2013.

7. Mohandas KM, Swaroop VS, Krishnamurthy S, Desai DC, Dhir V, Pradhan SA, Jagannath P and Desouza LJ: Unusual bone metastasis as the initial symptom of gastric cancer-a report of four cases. Indian J Cancer 30: 146-150, 1993.

8. Cao X, Cui F, Wei J, Wang Q, Deng LC, Liu BR and Shen WS Hearing loss due to metastasis of gastric cancer to temporal bone: A case report. Oncol Lett 11: 1305-1308, 2016.

9. Lim SM, Kim YN, Park KH, Kang B, Chon HJ, Kim C, Kim JH and Rha SY: Bone alkaline phosphatase as a surrogate marker of bone metastasis in gastric cancer patients. BMC Cancer 16: $385,2016$.

10. Silvestris N, Pantano F, Ibrahim T, Gamucci T, De Vita F, Di Palma T, Pedrazzoli P, Barni S, Bernardo A, Febbraro A, et al: Natural history of malignant bone disease in gastric cancer: Final results of a multicenter bone metastasis survey. PLoS One 8: e74402, 2013.
11. Mikami J, Kimura Y, Makari Y, Fujita J, Kishimoto T, Sawada G, Nakahira S, Nakata K, Tsujie M and Ohzato H: Clinical outcomes and prognostic factors for gastric cancer patients with bone metastasis. World J Surg Oncol 15: 8, 2017.

12. Ahn JB, Ha TK and Kwon SJ: Bone metastasis in gastric cancer patients. J Gastric Cancer 11: 38-45, 2011.

13. Riihimäki M, Hemminki A, Sundquist K, Sundquist J and Hemminki K: Metastatic spread in patients with gastric cancer. Oncotarget 7: 52307-52316, 2016.

14. Lee J, Lim T, Uhm JE, Park KW, Park SH, Lee SC, Park JO, Park YS, Lim HY, Sohn TS, et al: Prognostic model to predict survival following first-line chemotherapy in patients with metastatic gastric adenocarcinoma. Ann Oncol 18: 886-891, 2007.

15. Koizumi W, Narahara H, Hara T, Takagane A, Akiya T, Takagi M, Miyashita K, Nishizaki T, Kobayashi O, Takiyama W, et al: S-1 plus cisplatin versus S-1 alone for first-line treatment of advanced gastric cancer (SPIRITS trial): A phase III trial. Lancet Oncol 9: 215-221, 2008.

This work is licensed under a Creative Commons Attribution-NonCommercial-NoDerivatives 4.0 International (CC BY-NC-ND 4.0) License. 